\title{
Diacronie
}

Studi di Storia Contemporanea

$\mathrm{N}^{\circ} 34,2$ | 2018

Scuola e società in Italia e Spagna tra Ottocento e Novecento

\section{Rifrancesizzare i Valdostani}

Scuola e identità in Valle d'Aosta (1861-2017)

\section{Alessandro Celi}

\section{(2) OpenEdition}

\section{Journals}

\section{Edizione digitale}

URL: http://journals.openedition.org/diacronie/8111

DOI: $10.4000 /$ diacronie. 8111

ISSN: 2038-0925

Editore

Association culturelle Diacronie

\section{Notizia bibliografica digitale}

Alessandro Celi, « Rifrancesizzare i Valdostani », Diacronie [Online], Nㅜ 34, 2 | 2018, documento 2 Messo online il 29 juin 2018, consultato il 01 mai 2019. URL : http://journals.openedition.org/ diacronie/8111; DOI : 10.4000/diacronie.8111 


\title{
Diacronie
}

Studi di Storia Contemporanea

34, $2 / 2018$

Scuola e società in Italia e Spagna tra Ottocento e Novecento

\section{Rifrancesizzare i Valdostani. Scuola e identità in Valle d'Aosta (1861-2017)}

\author{
Alessandro CELI
}

Per citare questo articolo:

CELI, Alessandro, «Rifrancesizzare i Valdostani. Scuola e identità in Valle d'Aosta (1861-2017)», Diacronie. Studi di Storia Contemporanea : Scuola e società in Italia e Spagna tra Ottocento e Novecento, 34, 2/2018, 29/06/2018,

URL: <http://www.studistorici.com/2018/06/29/celi_numero_34/ >

Diacronie Studi di Storia Contemporanea $\rightarrow$ http://www.diacronie.it

Rivista storica online. Uscita trimestrale.

redazione.diacronie@hotmail.it

Comitato di direzione: Naor Ben-Yehoyada - João Fábio Bertonha - Christopher Denis-Delacour - Maximiliano Fuentes Codera Anders Granås Kjøstvedt - John Paul Newman - Deborah Paci - Niccolò Pianciola - Spyridon Ploumidis - Wilko Graf Von Hardenberg

Comitato di redazione: Jacopo Bassi - Luca Bufarale - Gianluca Canè - Fausto Pietrancosta - Alessandro Salvador - Matteo Tomasoni - Luca Zuccolo

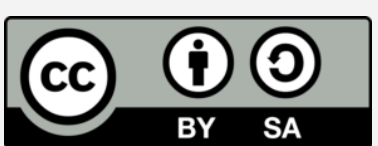

Diritti: gli articoli di Diacronie. Studi di Storia Contemporanea sono pubblicati sotto licenza Creative Commons 3.0. Possono essere riprodotti e modificati a patto di indicare eventuali modifiche dei contenuti, di riconoscere la paternità dell'opera e di condividerla allo stesso modo. La citazione di estratti è comunque sempre autorizzata, nei limiti previsti dalla legge. 


\title{
02/ Rifrancesizzare i Valdostani. Scuola e identità in Valle d'Aosta (1861-2017)
}

\author{
Alessandro CELI
}

L'articolo ripercorre le vicende politiche e legislative relative alla Scuola della Valle d'Aosta negli ultimi settant'anni. La Scuola fu scelta quale strumento per rifrancesizzare una popolazione che in seguito alle trasformazioni demografiche e alle politiche antifrancesi dello Stato liberale, prima, e del regime mussoliniano, poi, stava perdendo la tradizionale francofonia. Tale compito fu ostacolato dalle dinamiche della lotta politica locale, dalle resistenze dello Stato centrale e dalle scelte dei governi regionali. L'articolo analizza l'evoluzione delle norme che regolano l'organizzazione delle scuole della Valle d'Aosta in relazione all'evoluzione delle vicende politiche e sindacali locali e mette in particolare evidenza come le esigenze del consenso elettorale abbiano progressivamente svuotato la spinta ideale che aveva animato i dibattiti sulla Scuola tra gli anni Quaranta e Settanta.

In Valle d'Aosta, negli ultimi trent'anni le uniche manifestazioni pubbliche di protesta in grado di mobilitare centinaia di persone sono state quelle relative alla Scuola ${ }^{1}$. In una regione a lungo considerata ricca, con un tasso di disoccupazione estremamente basso e priva di una dialettica politica accesa, soltanto le questioni scolastiche hanno saputo suscitare una mobilitazione sociale degna di rilievo. Certamente, il fenomeno è dovuto anche ai numeri relativamente modesti della popolazione valdostana, che rendono il sistema scolastico il secondo settore per numero di addetti dopo l'impiego pubblico regionale, così come alla progressiva scomparsa del settore secondario in tutta la regione. Dalla seconda metà degli anni Settanta, infatti, la deindustrializzazione ha costituito il principale fenomeno economico locale, con una progressiva chiusura di tutte le industrie tessili ${ }^{2}$, la scomparsa di importanti insediamenti metallurgici ${ }^{3}$ e la massiccia riduzione di personale del polo siderurgico della Cogne, oggi ridotto a poco meno di un

\footnotetext{
${ }^{1}$ In particolare, le proteste contro la maturità bilingue nel 1998/9 e contro la Buona Scuola nel 2015 videro attuarsi numerose forme di protesta pubbliche (cortei, sit-in, presidi sotto il Palazzo regionale).

${ }^{2}$ Industrie tessili erano presenti a Verrès e Châtillon, dove lo stabilimento della Soie apparteneva a una delle maggiori società italiane del settore.

${ }^{3}$ L'ILLSA-VIOLA di Pont-Saint-Martin su tutte: in proposito, BARSIMI, Margherita (a cura di), Voci d'acciaio Storie di lavoro nelle testimonianze degli ex-dipendenti ILSSA-VIOLA, Musumeci, Saint-Christophe, 2017.
} 
migliaio di dipendenti, circa un decimo di quelli che contava negli anni di piena attività ${ }^{4}$. Se fattori esogeni hanno favorito la centralità della scuola come luogo di scontro e dibattito politico, sono, però, i fattori interni che hanno determinato tanta importanza e, in conseguenza, sancito la funzione di strumento di lotta politica assunto dalla scuola in Valle d'Aosta. Per comprendere l'importanza del sistema di istruzione ed educazione nell'attuale dibattito politico valdostano occorre, però, risalire alla metà dell'Ottocento e, più precisamente, alla proclamazione del Regno d'Italia.

\section{Prima del 1945}

Le condizioni con le quali si realizzò l'Unità della penisola e gli accordi internazionali con la Francia di Luigi Napoleone determinarono, tra le altre conseguenze, la scomparsa pressoché assoluta delle popolazioni di lingua francese all'interno dei domini di casa Savoia. Se fino al 1860 il $12 \%$ della popolazione del Regno di Sardegna era di madrelingua francese, l'anno successivo, con la cessione degli «Stati di là de' monti» al Secondo Impero, tale percentuale si era ridotta allo $0,2 \%$ e appariva insignificante rispetto ai milioni di nuovi sudditi acquisiti con la conquista dell'Italia meridionale. Le popolazioni che abitavano le alte valli del Pinerolese e di Susa e la Valle d'Aosta sembravano destinate a una rapida assimilazione, tanto per motivazioni economiche quanto demografiche. In realtà, la coscienza della propria diversità all'interno del nuovo Regno d'Italia e il desiderio di mantenere un'identità che era, nello stesso tempo, linguistica e religiosa consentirono ai Valdesi e ai Valdostani di contrastare con una certa efficacia la spinta all'uniformizzazione tipica dello Stato-nazione italiano. Un primo elemento da tenere in considerazione per comprendere i successivi sviluppi della scuola Valle d'Aosta è proprio quello religioso. Mentre nelle valli valdesi la lingua francese fu difesa in quanto fattore identitario legato alla predicazione del culto evangelico, in Valle d'Aosta essa divenne la bandiera di un clero maggioritariamente intransigente, geloso delle proprie prerogative e degli antichi privilegi della Chiesa gallicana, cui la diocesi di Aosta aveva aderito in quanto suffraganea dell'arcidiocesi di Tarantasia fino al 1860. Tra l'Unità e la Grande Guerra la lingua francese divenne così l'oggetto di una contesa in cui lo Stato liberale cercava di imporre l'insegnamento in lingua italiana attraverso la diffusione delle scuole pubbliche, mentre nelle scuole confessionali continuava l'uso del

\footnotetext{
${ }^{4}$ Sulla Società Cogne, BINEL, Corrado (a cura di), Dall'Ansaldo alla Cogne Un esempio di siderurgia integrale, 19171945, Milano, Electa, 1985; PEIRANO, Stefano, Operai in Cogne Dentro l'archivio di un grande stabilimenti siderurgico 1917-1942, Aosta, Istituto storico della Resistenza, 1997; MORETTO, Luca (a cura di), L'industria e la città : la Cogne e Aosta Storia di un secolo : architettura, siderurgia, territorio, Aosta, Tipografia Valdostana, 2002; COGNE ACCIAI SPECIALI, Cento anni di Cogne, Saint-Christophe, Duc, 2016. Sulle origini dello stabilimento, si veda anche CASTRONOVO, Valerio (a cura di), Storia dell'Ansaldo, vol. 4, L'Ansaldo e la Grande Guerra 1915-1918, Roma-Bari, Laterza, 1997.
} 
francese. In questo scontro giocò un ruolo importante anche il contesto geografico. La Valle d'Aosta è infatti una zona montuosa, in cui ogni singolo Comune comprende numerosi villaggi, all'epoca spesso isolati durante l'inverno a causa della neve. In essi l'insegnamento primario era assicurato dalle «écoles des hameaux», le scuole di villaggio nelle quali assolvevano il compito di maestro il vicario del parroco oppure una maestra, pagati direttamente dalla comunità, senza la mediazione degli organismi statali.

Tale situazione si mantenne pressoché invariata fino alla vigilia della Prima guerra mondiale, per essere poi sconvolta dai cambiamenti demografici indotti dal conflitto e, successivamente, dalla politica fascista sulla scuola, a partire dalla riforma Gentile. Poiché questo articolo intende presentare al lettore le vicissitudini della scuola valdostana del secondo dopoguerra, sarà sufficiente riassumere quanto avvenuto tra 1861 e 1945 dicendo che a partire dall'Unità d'Italia la scuola rappresentò l'epifenomeno del dibattito sulla lingua francese in Valle d'Aosta. Fu infatti intorno al diritto di mantenere l'insegnamento primario in tale lingua e alla possibilità di continuare ad insegnarla nel ciclo secondario che si sviluppò gran parte della dialettica tra lo Stato centrale, le autorità comunali e l'élite culturale locale. Un secondo elemento da ritenere è proprio quello della coesione dimostrata dalla classe dirigente valdostana, tanto laica quanto religiosa, nella difesa dell'uso del francese. Se le vicende risorgimentali e la successiva Questione romana divisero l'élite valdostana tra laici e sacerdoti, tra liberali e clericali, tra massoni e antimassoni, rimane però innegabile che tutti si adoperarono affinché la popolazione della Valle d'Aosta potesse continuare ad essere educata in lingua francese e tale lingua rimanesse quella della cultura del Paese, senza dimenticarne un uso pubblico che giungeva fino alla redazione dei verbali dei Consigli comunali ${ }^{6}$. Da questo retroterra, prima, durante e dopo la Seconda guerra mondiale ${ }^{7}$ sorsero le rivendicazioni che portarono, attraverso dinamiche non ancora del tutto

\footnotetext{
${ }^{5}$ Sulle vicende della Scuola valdostana in quei decenni, OMEZZOLI, Tullio, Lingua e politica nella provincia fascista: una antologia della stampa, una analisi della attività culturale fascista in Valle d'Aosta (1927-1945), Aosta, Musumeci, 1974; BÉTEMPS, Alexis, PRAZ, Vera, L'école d'autrefois en Vallée d'Aoste, Quart, Musumeci, 1984; CUAZ, Marco, Alle frontiere dello stato La scuola elementare in Valle d'Aosta, Milano, Franco Angeli, 1988; BERTOLINO, Fabrizio, REVELli, Luisa (a cura di), Pagine di storia valdostana, Aosta, Tipografia Valdostana, 2008; LOUVIN, Roberto, Autonomie, scuola e particolarismo linguistico in Valle d'Aosta, Torino, Libreria Stampatori, 2009; ID., La peculiarità della scuola valdostana tra Otto e Novecento L'istruzione come bene di comunità Spunti di riflessione dalla storia delle scuole di villaggio in Valle d'Aosta, in GENRE, Raimondo, TRON, Claudio (a cura di), Il sistema scolastico in Val Chisone prima e dopo l'unità d'Italia Dai conflitti alla convivenza, Perosa Argentina, LAR editore, 2012, pp. 33-50; CUAZ Marco La scuola elementare in Valle d'Aosta: acquisizioni, problemi e prospettive di ricerca, in PISERI, Maurizio (a cura di), L'alfabeto in montagna Scuola e alfabetismo nell'area alpina tra età moderna e XIX secolo, Milano, Franco Angeli, 2012; REINOTTI, Elio, L'istruzione elementare in Valle d'Aosta dal 1678 al 1822, Donnas, Biblioteca di Donnas, 2014.

${ }^{6}$ L'Archivio Storico Regionale conserva nel Fonds Ville i verbali del Consiglio comunale di Aosta, redatti in Francese fino al commissariamento deciso dal governo Mussolini nel 1923. In tale occasione, il commissario, pur riconoscendo che la lingua francese era quella prevalentemente parlata dalla popolazione, impose l'impiego dell'italiano «nel superiore interesse della nazione».

${ }^{7}$ Esemplari, in tal senso, gli scritti e l'azione di Emile Chanoux (1906-1944), martire della Resistenza, che maturò le proprie posizioni antifasciste negli anni Venti, proprio durante il dibattito sul ruolo delle scuole
} 
chiarite $^{8}$, al decreto legislativo luogotenenziale del 7 settembre 1945 n. 545 e al successivo Statuto di autonomia del 26 febbraio 1948. In entrambi i provvedimenti alla scuola era implicitamente assegnato il compito di rifrancesizzare una popolazione che, a partire dalla Grande Guerra, aveva visto un imponente cambiamento demografico. Tra il 1916 e il 1938, la Valle d'Aosta visse, infatti, tre grandi ondate migratorie. Fino al 1921 si assistette al massiccio arrivo di immigrati dalle altre regioni italiane, richiamati in Valle come operai per l'industria di guerra: questa prima ondata fu tanto importante che tra il censimento del 1911 e quello del 1921 la popolazione residente aumentò, benché più di 3500 persone, pari all'incirca al 5\% della popolazione del 1911, fossero morte in guerra o nell'epidemia di Spagnola seguita al conflitto. Tra il 1928 e il 1930 si assistette, invece, a un'ondata emigratoria che portò circa 20.000 valdostani a spostarsi nei paesi francofoni, soprattutto a Parigi e nelle zone agricole che circondano la capitale francese ${ }^{9}$, a seguito del fallimento del sistema creditizio cattolico locale ${ }^{10}$. Negli anni Trenta, invece, l'ampliamento dell'industria siderurgica per scopi bellici permise di colmare il vuoto causato dalla partenza dei Valdostani con alcune decine di migliaia di immigrati provenienti soprattutto dal Veneto ${ }^{11}$. Questi immigrati, facile preda della propaganda fascista, causarono una vera e propria spaccatura sociale e linguistica all'interno della Valle d'Aosta, la cui popolazione era ancora quasi totalmente francofona. Inoltre, i Veneti giunti nella regione molto spesso non parlavano neppure l'italiano, ma soltanto il dialetto della regione natia e per questo motivo costituivano una formidabile sfida pastorale per il clero diocesano. Quest'ultimo era cresciuto affiancando al latino del Seminario la lingua francese, sola lingua di cultura della Valle d'Aosta fino alla metà dell'Ottocento e, appunto, bandiera dell'intransigentismo cattolico locale e del rifiuto dell'Italia liberale da parte della Chiesa diocesana ${ }^{12}$. Quest'ultima introdusse, infatti, l'italiano come lingua di comunicazione nei corsi precedenti la Teologia solo negli anni Trenta, durante l'episcopato di mons. Francesco Imberti ${ }^{13}$. Durante la Grande guerra, i responsabili ecclesiali di Aosta si accorsero che la mancanza di

di villaggio e dell'uso della lingua francese nella Scuola della riforma Gentile. In merito si vedano gli scritti di Chanoux curati da Paolo Momigliano Levi: CHANOUX, Emile, Ecrits, URL: $<$ http://www.fondchanoux.org/lesecrits_1_0_523.aspx > [consultato il 31 maggio 2018].

${ }^{8}$ Sulle vicende successive alla Liberazione, CELI, Alessandro, «L'annessionismo valdostano tra censure, rimozioni e nuove ipotesi di ricerca», in Nazioni e regioni, 6, 2015, pp. 7-26; DÉSANDRÉ, Andrea, Sotto il segno del Leone, Aosta, Musumeci, 2015.

${ }^{9}$ CASTAGNARO, Cinzia, FRANCOVICH, Lisa, Evoluzione della popolazione e comportamenti demografici in Valle d'Aosta, Aosta, Fondation Emile Chanoux, 2004.

${ }^{10}$ Per una rapida sintesi sulla vicenda CELI, Alessandro, Il fallimento del Credit Valdôtain La fine dell'autonomia finanziaria valdostana, in Le Messager valdôtain 2011, Aosta, Imprimerie valdôtaine, Aosta 2010, pp. 77-79.

${ }^{11}$ In particolare da Belluno a Cogne, come descritto dalla tesi di Bruno Zanivan (ZANIVAN, Bruno, Cogne: miniera, lavoro, immigrazione Una comunità di alta montagna dagli anni '30 agli anni ' 60 , Tesi di laurea in scienze politiche e delle relazioni internazionali, Università della Valle d'Aosta/Université de la Vallée d'Aoste, a.a. 2013/2014) poi da Vicenza, Verona e Treviso.

${ }^{12}$ Significativamente, i soli deputati cattolici locali furono Edoardo Crotti di Costigliole, fondatore della Società della Gioventù Cattolica italiana, fino al 1871, e i fratelli Farinet a partire dal 1895.

${ }^{13}$ Sul prelato, CELI, Alessandro, I seicento giorni della diocesi di Aosta La Chiesa cattolica valdostana durante la Resistenza, Aosta, Le Château, 2008. 
sacerdoti e di suore in grado di parlare e ragionare come gli immigrati avrebbe reso questi ultimi facile preda della propaganda comunista, prima, e fascista, poi. Fu così che nell'estate del 1918 furono chiamati ad Aosta i Salesiani Don Bosco e le Figlie di Maria Ausiliatrice, con il precipuo incarico di assistere i figli e le figlie degli immigrati. Tale impegno si concretizzò nella costruzione dell'Istituto San Giovanni Bosco, comprensivo di un asilo e della scuola elementare, retto ancora oggi dalle Suore salesiane, che si affiancò alle scuole e agli asili gestiti dalla locale congregazione delle Suore di San Giuseppe ${ }^{14}$. Minor fortuna ebbe invece il ramo maschile, che soltanto nel 1948 riuscì ad aprire una propria scuola professionale a Châtillon, mentre ad Aosta il tentativo di costituire una comunità salesiana svanì all'inizio degli anni Venti e l'incarico di assistenza agli immigrati passò nel 1929 agli Oblati di Maria Immacolata, molti dei quali originari delle medesime zone di provenienza degli operai.

Tale situazione demografica, unita alle svariate ondate di gallofobia che caratterizzarono il regime fascista ${ }^{15}$, portò a un netto cambiamento nella formazione scolastica delle giovani generazioni, con i nati dopo il 1918 educati in una scuola primaria ormai pressoché italianizzata e, pertanto, non più in grado di leggere e parlare il francese. Per reazione, tutti i progetti politici espressi dai Valdostani durante la Resistenza rivendicarono il libero uso di questa lingua tanto nella Scuola quanto negli uffici pubblici. In particolare, nella Dichiarazione di Chivasso ${ }^{16}$ del 19 dicembre 1943, i rappresentanti della Valle d'Aosta e delle Valli valdesi chiedevano il «diritto all'insegnamento della lingua locale nelle scuole di ogni ordine e grado con le necessarie garanzie ai concorsi perché gli insegnanti risultino idonei a tale insegnamento», specificando, inoltre, che «l'insegnamento in genere sarà sottoposto al controllo ed alla direzione di un consiglio locale», al fine evidente di impedire eccessive ingerenze statali. Il concetto era ribadito, pur con finalità diverse, da Federico Chabod nel suo La Valle d'Aosta, l'Italia e la Francia del 27 settembre 1944:

Nelle scuole elementari e medie la lingua francese deve formare oggetto di apposito insegnamento, per ogni anno di corso e per un numero di ore settimanali pari a quello della lingua italiana. Inoltre, mentre l'insegnamento delle materie storico-letterarie (latino, greco,

\footnotetext{
${ }^{14}$ Questa Congregazione fu chiamata in Valle dal vescovo Agodino nel 1831.

${ }^{15}$ I governi liberale e mussoliniano furono particolarmente attenti all'uniformizzazione linguistica del Paese, come testimoniano tra l'altro, rapporti diplomatici del Consolato francese di Torino, che già nel 1919 e, poi, nel 1927 denunciavano i provvedimenti volti a cancellare l'uso del Francese nella scuola (Ministero Affari Esteri Francese, série Z carton 372, dossier n. 6, 1919-1929).

${ }^{16}$ Documento approvato a Chivasso dai rappresentanti delle Valle valdesi e della Valle d'Aosta, nel quale si auspicava la costituzione di un'Italia "economicamente ed amministrativamente...organizzat[a] con criteri federalistici" (il testo è riprodotto nel volume curato da Joseph-César Perrin: CHANOUX, Emile, De la "Déclaration de Chivasso» à «Federalismo ed Autonomie», Aosta, Imprimerie valdôtaine, 1973, p. 34). Per una presentazione generale del documento, MOMIGLIANO LEVI, Paolo, PERRIN, Joseph-César, Dichiarazione dei rappresentanti delle popolazioni alpine : Chivasso 19 dicembre 1943 : il contesto storico, i protagonisti e i testi, Aosta, Le Château, 2003.
} 
storia, geografia, filosofia, diritto) viene impartito in lingua italiana, l'insegnamento delle materie scientifiche (matematica, fisica, scienze naturali) viene impartito in lingua francese ${ }^{17}$.

I due documenti sono importanti perché mettono in rilievo i due aspetti che caratterizzeranno maggiormente le vicende scolastiche valdostane nei settant'anni successivi: la definizione dello stato giuridico dei docenti da parte di Stato e Regione e la presenza della lingua francese all'interno dei percorsi didattici.

\section{Le richieste del 1945 e le concessioni del 1948}

Alla Liberazione, le richieste dei Valdostani relativamente alla Scuola riguardarono, appunto, il ruolo della lingua francese nei percorsi scolastici e il controllo del personale docente. Data la situazione contingente, molte di esse furono inizialmente recepite. Il Decreto Luogotenenziale n. 545 del 7 settembre 1945 riconosceva, infatti, competenza amministrativa alla Valle anche per la Scuola elementare e media (art. 12 punto 4), con il relativo controllo sull'assunzione dei docenti come degli altri impiegati (art. 16 e 18). La medesima norma costituiva, però, un passo indietro rispetto alle richieste di Chabod dell'anno precedente, perché sanciva la parità di insegnamento tra italiano e francese solo per la lingua, mentre rimetteva a successivi accordi l'uso veicolare del secondo:

Nelle scuole di ogni ordine e grado esistenti nella Valle, all'insegnamento della lingua francese è dedicato un numero di ore settimanali pari a quello della lingua italiana. L'insegnamento di alcune materie può essere impartito in lingua francese. L'insegnamento delle varie materie è disciplinato dalle norme e dai programmi in vigore nello stato, con gli opportuni adattamenti alle necessità locali. Tali adattamenti, nonché la determinazione delle materie da insegnare in lingua francese, sono effettuati da commissioni miste, composte di rappresentanti del ministero della Pubblica Istruzione e di rappresentanti del Consiglio della Valle. Gli insegnanti delle scuole elementari e medie sono nominati dal Consiglio della Valle. Gli uni e gli altri devono possedere i titoli di studio prescritti dalle leggi dello stato; gli insegnanti delle scuole medie devono aver conseguito l'idoneità in un concorso generale per le scuole medie dello stato $^{18}$.

\footnotetext{
${ }^{17}$ Il documento è reperibile all'URL:

$<$ http://www.regione.vda.it/gestione/gestione_contenuti/allegato.asp?pk_allegato=127 > [consultato il 31 maggio 2018]. Sul tema si veda: NICCO, Roberto, La Resistenza in Valle d'Aosta, Aosta, Musumeci,1995, p. 188. ${ }^{18}$ Decreto legislativo luogotenenziale 7 settembre 1945 n. 545, art. 18 (Gazzetta Ufficiale, 20 settembre 1945, n. 113).
} 
La riduzione a possibilità di quanto Chabod aveva proposto come obbligo e la subordinazione di tale possibilità ad un accordo con lo Stato, così come la specificazione che i docenti di Scuola media dovevano essere selezionati da questo e non dall'ente locale, furono l'origine dei problemi che alimentarono il dibattito sulla Scuola in Valle nei successivi trent'anni. Gli articoli 39 e 40 dello Statuto di Autonomia ${ }^{19}$ ripresero, infatti, in maniera integrale il Decreto Luogotenenziale e rimandarono le decisioni sulla Scuola a trattative che, come per altri, importanti aspetti dello Statuto, stentarono a concretizzarsi ${ }^{20}$.

\section{Primo problema: il francese lingua strumentale}

Il primo problema a non essere risolto fu quello dell'uso del francese come lingua d'insegnamento: dopo le proposte di Chabod, che prevedevano l'insegnamento in lingua francese delle materie scientifiche, seguirono quelle del primo Consiglio regionale, che il $1^{\circ}$ agosto 1946 raccomandava di svolgere in lingua francese l'insegnamento della religione, della storia e della geografia locali. A questa prima ipotesi fece seguito, l'11 novembre successivo, una seconda proposta, che assegnava l'insegnamento in questa lingua anche a matematica e scienze in terza, quarta e quinta elementare. L'ondeggiamento nella scelta delle materie rivela la differenza delle posizioni del dibattito in atto e gli obiettivi ad esso sottesi: organizzare una scuola nella quale la lingua francese avesse avuto ampio spazio avrebbe significato formare nuove generazioni in grado di comprendere e parlare tale lingua. Tale prospettiva assumeva una valenza politica fondamentale, in una Valle percorsa da spinte separatiste, in cui lo scontro politico rischiava di sfociare in aperta rivolta tra sostenitori dell'unità dello Stato e fautori della secessione a favore della Francia.

Il successivo riconoscimento ufficiale del bilinguismo perfetto da parte dello Statuto di Autonomia del 28 febbraio 1948 sancì, almeno formalmente, la soluzione del dibattito sulle lingue dei Valdostani, ma esso fu immediatamente messo in discussione da quanti sostenevano che si trattava di un'endurmia, un provvedimento concesso a malincuore dalla Repubblica per placare le rivendicazioni dei Valdostani, senza però dare loro quanto essi realmente desideravano. In effetti, lo Statuto, stabilendo la parità tra le due lingue in un contesto ormai profondamente italianizzato, soprattutto nei centri industriali, poneva la seconda lingua in posizione di inferiorità, in quanto il

\footnotetext{
${ }^{19}$ Legge costituzionale n. 4 del 26 febbraio 1948 (Gazzetta Ufficiale, 10 marzo 1948).

${ }^{20}$ L'esempio più rilevante della resistenza degli Organi dello Stato nei confronti dello Statuto di autonomia è dato dalla mancata realizzazione della Zona Franca, prevista dall'art. 14 dello Statuto, ma subordinata a «modalità d'attuazione...concordate con la Regione e stabilite con legge dello Stato», legge che non vide mai la luce.
} 
suo uso quotidiano era relegato a contesti sempre più minoritari, come l'ecclesiale, dove comunque il clero stava progressivamente trascurando l'uso del francese nella predicazione ${ }^{21}$.

Contemporaneamente, la lingua d'oltralpe divenne un elemento centrale nel dibattito politico: il movimento dell'Union Valdôtaine ${ }^{22}$ ne rivendicava l'uso esclusivo nella Regione e contestava una scuola in cui essa era di fatto relegata al ruolo di lingua straniera. Tale, fu, infatti, l'esito del dibattito dei primi anni del Dopoguerra: il francese fu insegnato in quanto lingua per un numero di ore pari all'italiano, ma come fosse una lingua straniera. Soltanto dagli anni Settanta fu utilizzata come lingua strumentale per illustrare i contenuti di altre discipline. Tale cambiamento fu possibile a seguito di due avvenimenti concomitanti. Da un lato, l'Union Valdôtaine aveva raggiunto l'egemonia nel contesto politico locale e poteva quindi influenzare maggiormente le scelte in campo scolastico. Dall'altro, gli esiti della protesta sessantottina portarono anche allo sviluppo di una nuova sensibilità verso il dialetto ${ }^{23}$, mentre nuove impostazioni pedagogiche si diffondevano soprattutto tra le insegnanti più giovani, che arricchirono l'insegnamento in lingua francese con l'applicazione delle teorie di Célestin Freinet, unendo così il rinnovamento didattico alla promozione dell'uso di questa lingua ${ }^{24}$.

\section{Secondo problema: lo stato giuridico dei docenti}

La necessità di insegnare la lingua francese per un numero di ore pari a quella italiana ebbe come conseguenza l'aumento delle ore di lezione nella scuola elementare, che passarono a trenta rispetto alle venticinque previste all'epoca. Questa esigenza provocò un corrispondente aumento del carico di servizio degli insegnanti che già il 13 febbraio 1947, con la delibera n. 10 del Consiglio

\footnotetext{
${ }^{21}$ Sull'evoluzione linguistica in Valle d'Aosta FONDATION EMILE CHANOUX, Une Vallée d'Aoste bilingue dans une Europe plurilingue, Aosta, Tipografia valdostana, 2003. Per una riflessione sulla necessità di una discriminazione positiva a favore delle minoranze linguistiche, LATTANZI, Giorgio, La tutela dei diritti delle minoranze in Italia, URL:

< www.cortecostituzionale.it/documenti/relazioni_internazionali/RI_20130606_LATTANZI.pdf > [consultato il 31 maggio 2018].

${ }^{22}$ Per la storia dell'Union Valdôtaine: BOIARDI, Franco, DI CAPUA, Giovanni, Grande enciclopedia della politica I protagonisti dell'Italia democratica. L'Union valdôtaine, 1943/1993, Roma, Ebe, 1994. Per l'analisi delle vicende dell'immediato dopoguerra, oltre alle già citate opere di Celi e Désandré, si veda TREVES, Vincent, Entre l'histoire et la vie, Aoste, Le Château, 1999.

${ }^{23}$ Sulle origini teoriche e lo sviluppo dell'ideologia che contrapponeva il patois locale a italiano e francese: CELI, Alessandro, «Harpitanya e le lingue dei Valdostani», in BENELLI, Graziano, SAGGIOMO, Carmen (a cura di), Un coup de dés 2, Napoli, Edizioni Scientifiche Italiane, 2014, pp. 63-79. Sui Centres culturels, culla della riflessione sul francoprovenzale: ARMAND, Henri, Le Centre Culturel et la Bibliothèque, in PERRIN, JosephCésar (coord.), Saint-Nicolas Histoire et culture dans un pays de montagne, Saint-Christophe, Duc 2017, pp. 237244.

${ }^{24}$ In proposito, $I X^{e}$ stage international de l'école moderne pédagogie Freinet: Champorcher Vallée d'Aoste 26-31 aout 1969, s.l., [1969?]; DECIME, Rita (coord.), L'éducation bilingue précoce Aspects socio-psychologiques et institutionnels Actes du colloque international organisé par l'Assessorat régional à l'Instruction publique de la Vallée d'Aoste Saint-Vincent 18-19-20 juin 1984, Quart, Musumeci, 1986.
} 
regionale, ottennero un aumento della retribuzione in ragione delle ore aggiuntive di insegnamento prestate.

Fu l'inizio di un rapporto privilegiato tra scuola primaria e potere politico locale, che vedeva le maestre godere di un reddito nettamente superiore a quello delle loro colleghe in servizio nelle altre zone d'Italia e svolgere, indirettamente, un capillare ruolo di propaganda in tutti i Comuni.

Prove evidenti di tale connubio furono i mutamenti nell'adesione sindacale nel settore primario; la continua attenzione dimostrata dal mondo politico verso quest'ordine di scuola, sul quale lo Statuto di autonomia concedeva maggiori competenze rispetto alle superiori ${ }^{25}$ e, quindi, maggior possibilità di intervento; le pagine dedicate alle maestre sul «Messager Valdôtain», l'annuale almanacco cattolico che dal 1912 conserva la memoria dei principali avvenimenti valdostani dell'anno trascorso.

Il «Messager» ben rappresenta i cambiamenti susseguitisi nel tempo: fino al 1964, le sue pagine riportavano il nome di ogni insegnante di scuola primaria, suddivise per Comune di servizio. Si trattava del riconoscimento implicito del ruolo delle maestre in una Valle in cui la Scuola primaria era, fino a quel momento, sottoposta all'egemonia culturale cattolica, direttamente nelle scuole private gestite dalle congregazioni religiose, indirettamente in quelle statali, dove era maggioritario il peso sindacale del Sindacato Scuola Elementare (Sinascel) della Cisl.

Col tempo, però, tale egemonia cominciò a scemare ed è significativo che proprio dal 1965, dopo cinque anni di governo regionale a guida comunista e autonomista ${ }^{26}$, l'almanacco abbia smesso di indicare il nome di tutte le maestre. Contestualmente avevano cominciato la loro ascesa le organizzazioni sindacali della Scuola che avevano in PCI e UV i loro partiti di riferimento. Il sorpasso definitivo avvenne negli anni Ottanta, quando il Sinascel-Cisl perse parte delle proprie iscritte in seguito a una scissione interna, sopraggiunta dopo la spaccatura della DC locale e la nascita di un secondo partito cattolico. Le maestre fuoriuscite dal sindacato bianco confluirono nello Snals, il sindacato autonomo che aveva raccolto l'esperienza di diverse organizzazioni sindacali, di area cattolica e di centro-destra, mentre Cgil e Savt (il sindacato autonomista collaterale all'Union Valdôtaine) approfittavano della situazione per erodere ulteriormente i consensi verso la Cisl.

Il mutamento nel panorama sindacale fu tra le cause delle dinamiche relative al dibattito sullo stato giuridico del personale scolastico. In base alle proposte dell'immediato dopoguerra, insegnanti e docenti delle scuole regionali avrebbero dovuto dipendere dalla Regione Autonoma. Tale proposta trovò un riconoscimento normativo col Decreto Legislativo del Capo provvisorio

${ }^{25}$ L'art. 3 dello Statuto concede alla Regione potestà legislativa «di integrazione e di attuazione» sull'istruzione materna, elementare e media, mentre l'art. 2 concede quella concorrente nell'istruzione tecnico-professionale.

${ }^{26}$ Nel 1959, dopo una legislatura che aveva visto la DC governare da sola grazie a un sistema elettorale maggioritario, l'alleanza tra Union Valdôtaine e partiti di sinistra relegò lo Scudocrociato all'opposizione. 
dello Stato n. 365 dell'11 novembre 1946, che però non venne mai applicato, cosicché il personale insegnante rimase formalmente dipendente dallo Stato, ma in servizio presso scuole appartenenti alla Regione e, per quanto riguarda la scuola elementare, con obblighi e trattamento economici diversi rispetto a quelli statali. Il governo regionale a guida democristiana, durante la II legislatura regionale (1954-59), tentò di risolvere il problema, proponendo una legge regionale che istituiva un "ruolo statale speciale per la Valle d'Aosta», approvata dal Consiglio con l'astensione dell'intera opposizione. La norma fu, però, rinviata dalla Commissione di coordinamento, l'organismo paritetico tra Stato e Regione che doveva esprimersi sulla compatibilità tra normativa regionale e leggi statali. La bocciatura dimostrò che lo Stato italiano non intendeva concedere ulteriori spazi decisionali alla Regione, benché quest'ultima stesse operando soltanto per applicare un diritto sancito dalle leggi approvate dal medesimo Stato. Significativo, in tal senso, il fatto che l'allora Presidente della Regione, il democristiano Vittorino Bondaz, avesse assicurato in aula che il disegno di legge era "stato predisposto... in seguito a contatti avuti con il Ministro della Pubblica Istruzione On.le Moro, fin dalla scorsa primavera» ${ }^{27}$, contatti che evidentemente non furono sufficienti ad assicurare l'approvazione della legge. Questa fu dunque rinviata al Consiglio regionale e poté essere approvata ${ }^{28}$ soltanto con una sostanziale modifica: anziché prevedere la costituzione di un «ruolo speciale», essa stabilì soltanto l'istituzione di un «albo speciale». In pratica, i docenti in servizio nelle Scuole della Valle risultavano formalmente titolari di una cattedra in un'altra località d'Italia, ma in servizio in Valle come insegnanti comandati, una situazione di continua incertezza che rendeva anch'essa evidente l'impatto delle decisioni statali nel limitare l'autonomia che lo stesso Stato aveva concesso.

Un esito tanto riduttivo fu dovuto non solo alle resistenze del centralismo romano, ma anche alle dinamiche della lotta politica valdostana. La norma fu definitivamente approvata a poche settimane dalle elezioni che il 17 maggio successivo avrebbero relegato all'opposizione la Democrazia Cristiana dopo dieci anni di egemonia a Palazzo regionale. L'imminenza del voto aveva trasformato l'approvazione della norma in un elemento di lotta politica in cui i partiti all'opposizione - e i sindacati ad essi correlati - poterono esercitare tutte le loro critiche a fini propagandistici. Quest'ultimo elemento rappresenta un'ulteriore costante nelle vicende della Scuola valdostana: a fronte di provvedimenti utili, se non necessari, le logiche della lotta politica locale prevalevano, anche a scapito dell'intero sistema scolastico e, più in generale, dell'intera popolazione valdostana, mentre gli organi dello Stato, tanto legislativi quanto esecutivi, non perdevano occasione per erodere le competenze statutarie.

\footnotetext{
${ }^{27}$ Il verbale della riunione è pubblicato nel volume edito dalla Presidenza del Consiglio regionale Dibattiti sull'Autonomia La Scuola, Aosta, Tipografia valdostana, 1988, p. 94.

${ }^{28}$ Legge regionale n. 3 del 21 aprile 1959 Norme per l'ordinamento delle scuole materne, elementari e medie e del relativo personale (Bollettino Ufficiale della Regione Valle d'Aosta 30 aprile 1959).
} 
A dimostrazione di questa affermazione è possibile citare il Disegno di legge presentato alla Camera dai deputati Tristano Codignola, responsabile scuola del PSI, e Luigi Borghi, democristiano, che il 18 maggio 1965 firmarono la proposta di abrogare il Decreto Legislativo del 1946. In quel momento, la Regione autonoma era ancora guidata da un governo che vedeva alleati autonomisti, socialisti e comunisti, mentre a Roma era ormai consolidato il centro-sinistra con il PSI alleato alla DC. L'iniziativa dei due parlamentari rappresentava, quindi, un evidente tentativo di porre in crisi l'alleanza tra PSI e PCI, poiché contraddiceva quanto proposto dalla DC nella legislatura precedente, ma era ora utile allo Scudocrociato per spingere il PSI valdostano dalla propria parte. Non a caso, nel successivo dibattito sul DdL in Consiglio regionale, i consiglieri liberali, alleati della DC, si schierarono apertamente a favore del provvedimento, motivandolo con l'interesse superiore degli studenti, che cambiavano ogni anno insegnanti a causa dell'aleatorietà delle nomine sulle numerose cattedre vacanti ${ }^{29}$. Tale motivo corrispondeva a un dato reale: ancora oggi, infatti, una percentuale importante del personale docente della Valle d'Aosta è precario, ma la soluzione proposta all'epoca ledeva pesantemente le competenze statutarie e fu pertanto osteggiata dalla maggioranza consiliare e dai rappresentanti valdostani in Parlamento, che riuscirono a impedire l'approvazione del provvedimento al Senato.

L'episodio dimostra come la politica scolastica in Valle d'Aosta sia spesso ridotta a strumento per scopi che poco o nulla hanno a che fare con l'istruzione. Non a caso, il successivo provvedimento che interessò la Scuola fu la legge regionale 2 febbraio 1968, n. 1. Si trattava di una norma che riformava la precedente disciplina sulle indennità per l'aumento orario nella Scuola elementare, risalente al 1947, recando ulteriori vantaggi economici alle maestre, incluso la pensionabilità dell'indennità di francese. Anche in questo caso, occorre mettere in relazione il provvedimento con il contesto politico coevo: dalla metà del 1967, la DC era tornata alla guida della Regione dopo otto anni di opposizione e la nuova legge costituiva un'occasione per accrescere il consenso in favore del governo in previsione delle elezioni regionali del 21 aprile successivo.

Il problema dello stato giuridico dovette attendere ancora qualche anno per giungere a una prima, solida soluzione: il Decreto del Presidente della Repubblica n. 861 del 31 ottobre 1975 istituì infine i ruoli regionali e aprì la strada alle successive norme che permisero di definire il ruolo del francese come lingua veicolare, l'art. 28 della legge 16 maggio 1978 n.196 e l'art. 21 della legge regionale 15 giugno 1983 n. $57^{30}$.

Anche in questo caso è possibile mettere in relazione il contesto sociopolitico del momento e $\mathrm{i}$ cambiamenti in ambito scolastico. In particolare, il cambiamento a livello locale fu favorito da due fattori: le difficoltà del governo centrale, nel pieno della crisi degli anni Settanta che aveva

\footnotetext{
${ }^{29}$ Verbale dell'adunanza del 15 marzo 1967 in Dibattiti sull'Autonomia, cit., pp. 140 et seq.

${ }^{30}$ Bollettino Ufficiale della Regione Valle d'Aosta, 20 giugno 1983.
} 
portato anche all'approvazione dei Decreti Delegati, e le conseguenze dei cambiamenti politici a livello locale, con l'inizio dell'ascesa dell'Union Valdôtaine dopo la nascita di un secondo partito cattolico a seguito della scissione della DC.

Esiste poi un ulteriore elemento da mettere in evidenza, un elemento che costituisce la terza costante nelle vicende della Scuola in Valle d'Aosta: i maggiori cambiamenti a livello locale avvennero sempre in parallelo con provvedimenti sulla Scuola a livello italiano, talvolta anticipandoli di qualche mese, talvolta seguendoli, anche in forma ampliata.

\section{Sperimentazioni e anticipazioni}

Il DPR 861/75 rappresentò una svolta nella Scuola della Valle d'Aosta, favorendo lo sviluppo di sperimentazioni che, per alcuni anni, posero il sistema scolastico valdostano all'avanguardia nel panorama nazionale. Già in precedenza la Valle si era segnalata per l'attivazione del Biennio Unitario Sperimentale nella Scuola superiore, uno dei fiori all'occhiello della Giunta demopopolare guidata da César Dujany (1970-74). Si trattava del tentativo di uniformare i primi due anni del Secondario Superiore in un unico percorso propedeutico alla successiva specializzazione nei diversi indirizzi ${ }^{31}$. La sperimentazione non sopravvisse alla caduta del Governo Dujany, ma rimane significativa per aver precorso i tempi in una Regione che, fino a quel momento, non aveva dato segni di particolari spinte innovatrici.

In realtà, proprio in quegli anni la Valle vedeva ravvivarsi il dibattito sull'identità dei Valdostani, sulle loro lingue e sui mezzi per salvaguardarle: la Giunta Andrione, che sostituì quella Dujany, ebbe come assessore all'Istruzione la professoressa Maria Ida Viglino, già a capo del locale Comitato di Liberazione Nazionale negli ultimi mesi di guerra ${ }^{32}$. La docente incarnò il desiderio di rinnovamento della Scuola in chiave identitaria, ma inclusiva, e fu l'anima della nuova Scuola Materna regionale, che costituì il fiore all'occhiello del governo unionista ${ }^{33}$. Il nuovo grado di scuola permetteva, infatti, di intraprendere con rinnovato vigore l'opera di rifrancesizzazione, di fatto incompiuta per le difficoltà normative che avevano rallentato l'azione della Regione Autonoma. Il progetto promosso dall'assessore Viglino prevedeva lo sviluppo di una scuola perfettamente bilingue, nella quale i bambini fossero inseriti in percorsi scolastici nei quali il francese era lingua veicolare per metà delle ore settimanali di lezioni. A supporto di questo

\footnotetext{
${ }^{31} \mathrm{Fu}$ autorizzato dal Decreto Ministeriale del 31 luglio 1970.

${ }^{32}$ La Viglino, rappresentante del PSI col nome di battaglia Piera, fu nominata Presidente del CLN aostano il 24 gennaio 1945 e in questa veste fu tra i protagonisti della liberazione di Aosta nell'aprile successivo. In proposito, CUAZ, Marco, MOMIGLIANO LEVI, Paolo, RICCARAND, Elio (a cura di), Cronologia della Valle d'Aosta 1848-2000, Aosta, Stylos, 2003, p. 387; NICCO, Roberto, Maria Ida Viglino, in Les Cent du Millénaire, Quart, Musumeci, 2000, p. 309.

${ }^{33}$ Benché fosse stata istituita durante il governo Dujany, nel 1972.
} 
approccio didattico fu organizzata una vasta formazione delle insegnanti, con periodi di permanenza presso Scuole e Università francofone di Belgio, Francia e Svizzera. In tal modo si ottenevano risultati su più livelli: sul piano didattico, le insegnanti valdostane entrarono in contatto con le teorie e i metodi didattici più recenti, rinnovando così l'approccio alla professione; su quello linguistico, il soggiorno all'estero migliorò le competenze delle maestre; l'organizzazione didattica della Scuola bilingue richiese poi un aumento dell'organico, mentre la generalizzazione della Scuola materna regionale sottrasse l'infanzia al controllo cattolico, egemone negli asili privati, e portò sotto il controllo della Regione gli asili (e le maestre) delle soppresse scuole materne comunali di Aosta. In tal modo, la Scuola materna diventò uno strumento di consenso e di propaganda e, ancora una volta, il mondo dell'educazione risultò funzionale alla lotta politica locale.

La medesima strategia fu alla base della riforma della Scuola primaria regionale, che nel 1988 anticipò di un anno la struttura statale basata su moduli di tre maestri su due classi, introdotta con la legge 5 giugno 1990 n. 148. La Regione Valle d'Aosta adottò tale organizzazione, che fece aumentare del 50\% l'organico docente del Settore primario, giustificandolo con l'adozione di una didattica che, come nella Materna, prevedeva metà delle ore di insegnamento in francese per tutte le discipline.

A differenza di quanto avvenuto dieci anni prima nella Materna, però, la spinta ideale che aveva animato la nascita di quel grado di Scuola era venuto meno. Maria Ida Viglino era scomparsa, il Presidente Andrione aveva rassegnato le dimissioni per problemi giudiziari e la nuova Giunta, guidata da Augusto Rollandin, non sembrava prestare la medesima attenzione alla Scuola. Così, dopo alcuni anni, le occasioni di formazione all'estero si diradarono, mentre scomparve del tutto il controllo sulla reale presenza del francese nella didattica quotidiana da parte degli ispettori scolastici regionali, lasciando alla libera iniziativa dei singoli gruppi-docenti l'applicazione di un obbligo di legge.

Esemplare, in tal senso, fu il successivo provvedimento che riguardò la Scuola valdostana ossia l'introduzione dei progetti bilingui nella Scuola Media con la delibera di Giunta del 22 luglio 1994 n. 5884 e la successiva legge regionale 22 agosto 1994 n. $53^{34}$. Si trattò, nuovamente, di provvedimenti assunti sulla scia di una riforma a livello statale, in questo caso l'introduzione obbligatoria dell'insegnamento della lingua inglese nella Media. Per adeguare la Scuola valdostana alla riforma, tenuto conto dell'obbligo statutario di svolgere un numero di ore in lingua francese pari a quelle previste per la lingua italiana, la Giunta regionale guidata da Dino Viérin stabilì, con un Decreto del Presidente della Giunta, che nella Scuola Media le lezioni si sarebbero svolte in moduli orari di 50 minuti anziché in ore da 60 , così da ottenere 36 moduli mattutini articolati in

\footnotetext{
${ }^{34}$ Bollettino Ufficiale Regione Valle d'Aosta, 30 agosto 1994.
} 
sei giorni di lezione alla settimana. In questi moduli veniva lasciata ai docenti la libertà di svolgere ore in compresenza con i colleghi, durante le quali attuare attività in lingua francese. Si trattava, però, di un approccio didattico diverso rispetto a quello della Scuola Materna e di quella Primaria, i cui reali effetti sulla preparazione degli studenti non sono mai stati valutati pubblicamente, tenuto conto della diminuzione del tempo dedicato a ogni disciplina ${ }^{35}$.

\section{La maturità bilingue}

Il fallimento del progetto impostato da Maria Ida Viglino fu provato anche dalla vicenda della cosiddetta «maturità bilingue». In occasione della riforma dell'Esame di Stato prevista dalla legge 425 del 1997, la Regione introdusse una quarta prova scritta obbligatoria per tutti, da svolgere in lingua francese ${ }^{36}$. Il provvedimento provocò accese proteste, perché oggettivamente negativo per gli studenti. In base alla legge regionale, infatti, il voto della quarta prova viene calcolato insieme a quello dello scritto di italiano per formare una media che contribuisce poi alla formulazione del voto finale. In tal modo, però, se la differenza di valutazione delle due prove supera il punto ${ }^{37}$, come avviene nella maggior parte dei casi, la loro media risulta penalizzante e dà origine a un voto inferiore a quello degli studenti che sono sottoposti a sole tre prove scritte.

Le proteste di docenti e studenti riempirono le strade di Aosta per alcuni mesi tra il 1998 e il 1999, ma non servirono a far recedere il Consiglio regionale dalle proprie posizioni. Si palesò, così, una quarta costante nelle vicende scolastiche regionali: il partito autonomista, ormai egemone a livello politico, sceglieva la via amministrativa e legislativa per imporre scelte non condivise da parte della popolazione, rinunciando, però, a convincerla delle proprie ragioni.

Si realizzò, quindi, una singolare frattura: mentre il consenso elettorale per l'Union Valdôtaine continuava a crescere, veniva meno l'adesione agli ideali che a cavallo della Seconda guerra mondiale, avevano ispirato la nascita del movimento. Un processo che avrebbe portato alla crisi dell'autonomismo valdostano meno di dieci anni dopo, quando, dopo aver raggiunto la maggioranza assoluta dei seggi in Consiglio regionale nelle elezioni regionali del 2008, l'Union Valdôtaine iniziò un processo disgregativo contrassegnato dalla fuoriuscita di iscritti che diedero vita a tre altri movimenti autonomisti, esplicitamente alternativi all'UV ${ }^{38}$.

\footnotetext{
${ }^{35}$ A titolo di esempio, 6 moduli settimanali di italiano corrispondono a 5 ore di insegnamento, con una riduzione del monte-ore disciplinare corrispondente a quasi sei settimane per ciascun anno scolastico.

${ }^{36}$ La prova è disciplinata dalla legge regionale 3 novembre 1998 n. 52 (Bollettino Ufficiale 10 novembre 1998) e dal DPR 7 gennaio 1999 n.13.

${ }^{37}$ La norma prevede che le frazioni di mezzo punto, derivanti dalla media dei due voti, siano arrotondate all'unità superiore. È evidente che se la differenza tra il voto dello scritto di italiano e il voto di francese è di due punti o più, la media che risulta diminuisce il voto più alto conseguito, nella maggior parte dei casi quello di italiano (da qui, l'origine delle proteste).

${ }^{35}$ Nel 2012 Laurent Viérin, all'epoca assessore all'Istruzione e Cultura, abbandonò l'Union per fondare
} 


\section{L'autonomia scolastica e le riforme degli anni Duemila}

La dinamica emersa nel 1998 si accentuò negli anni successivi: mentre le difficoltà politiche imponevano cambi di governo abbastanza frequenti ${ }^{39}$, la necessità di adeguare la normativa regionale alle diverse riforme che a livello nazionali si susseguirono a partire dall'introduzione dell'autonomia scolastica (DPR 275/1999) portò a una serie di provvedimenti - le leggi regionali 19/2000, 18/2005 e 18/2016 - che non modificarono le prospettive di sviluppo precedenti. Ogni provvedimento legislativo mirò, infatti, più alla salvaguardia o all'ampliamento degli organici che all'affermazione di una scuola francofona. Prevaleva, così, la ricerca del consenso tramite il controllo delle assunzioni rispetto allo sviluppo di un sistema di istruzione realmente francofono. Questa scelta accentuò il disinteresse per la didattica in francese, soprattutto da parte delle insegnanti più giovani, quelle che, per motivi anagrafici, non avevano conosciuto Maria Ida Viglino né avevano frequentato corsi all'estero. A tal proposito, è opportuno segnalare un analogo fenomeno, che ha riguardato la formazione dei nuovi insegnanti delle scuole primaria e dell'infanzia da parte dell'Università della Valle d'Aosta.

L'istituzione di un centro studi di livello universitario in Valle era stata a lungo vagheggiata da parte degli esponenti politici locali più attenti alla cultura: negli anni Sessanta, una prima risposta fu portata con la creazione del Collège d'Études fédéralistes da parte degli unionisti Corrado Gex e Mario Andrione ${ }^{40}$, mentre nel decennio successivo fu il governo demopopolare a ipotizzare la trasformazione della caserma Testafochi di Aosta in polo universitario e a favorire la riflessione in merito ${ }^{41}$.

L'Università della Valle d'Aosta divenne realtà quando la legge Bassanini, n. 59 del 15 marzo 1997, autorizzò la Provincia autonoma di Bolzano e la Regione Valle d'Aosta ad aprire un'università pubblica non statale. Il progetto dell'epoca, ampiamente pubblicizzato dai giornali, prevedeva l'apertura del corso di laurea in Scienze della Formazione Primaria, destinato alla preparazione di insegnanti per la Scuola primaria e dell'infanzia, nel quale ampio spazio doveva

l'Union Valdôtaine Progressiste. Successivamente l'ex Presidente della Regione, Luciano Caveri, creò il Mouv e l'ex senatore e assessore regionale alla Sanità, Antonio Fosson, il movimento Pour Notre Vallée. Alle tre compagini occorre aggiungere anche l'ALPE-Autonomie, Liberté, Participation, Ecologie, nato dal 2010 dalla confluenza dei Verdi alternativi con altri due movimenti autonomisti, conseguenza di precedenti scissioni dell'UV.

${ }^{39}$ Nelle ultime quattro legislature, dal 2002 al 2018, si sono succeduti sette Presidenti della Giunta.

${ }^{40} \mathrm{Fu}$ fondato nel 1961. Per una sua breve storia, URL: < http://fondchanoux.org/college.aspx > [consultato il 31 maggio 2018].

${ }^{41}$ In proposito: OMEZZOLI, Tullio «Quelques anticipations sur la nouvelle structure universitaire valdôtaine», in Bullettin de l'Académie Saint-Anselme, XLV, 1972, pp. 141-152. 
essere destinato all'insegnamento in lingua francese e alla frequenza in università francofone, così da consentire il riconoscimento del titolo anche in Belgio e Francia ${ }^{42}$.

In realtà, i periodi di frequenza all'estero previsti dall'ordinamento del corso di laurea furono soltanto facoltativi ${ }^{43}$ e gli insegnamenti in lingua francese si ridussero progressivamente nel corso degli anni ${ }^{44}$. Analizzando nello specifico i singoli insegnamenti, occorre poi rilevare che non fu mai prevista esplicitamente la preparazione bilingue in tutte le materie, pur essendo sancito il bilinguismo perfetto nella Scuola primaria ossia l'obbligo per gli insegnanti di svolgere in francese la metà delle ore in ogni disciplina (per la quale avrebbero dovuto, quindi, ricevere adeguata preparazione). Proprio questo particolare dimostra l'apatia nei confronti del francese da parte delle istituzioni e della politica locale per i primi tre lustri del nuovo secolo, durante i quali, almeno in apparenza, fu riservata maggiore attenzione all'insegnamento del patois, culminata nel tentativo di introdurlo come materia obbligatoria nella Scuola primaria ${ }^{45}$.

Soltanto nel 2015, a seguito dell'approvazione della legge 107 a livello nazionale, l'Amministrazione regionale intraprese un generale piano di riforma del sistema scolastico locale ${ }^{46}$. Esso fu incentrato sull'ampliamento delle ore in francese veicolare nella Scuola media e superiore, su una maggior presenza della lingua inglese in tutto il ciclo scolastico, sulla base di un progetto plurilingue sviluppato in precedenza nella Provincia autonoma di Trento ${ }^{47}$, e sul sostanzioso aumento dell'insegnamento in francese nel corso di laurea in Scienze della formazione primaria, a seguito della constatata inadeguatezza dei precedenti ordinamenti.

Il tentativo di riforma ha, però, visto la dura opposizione tanto dei docenti, quanto dei sindacati e dell'opinione pubblica. Infatti, oltre al generale malcontento suscitato dalla Buona Scuola a livello statale, il provvedimento ha scontato sia la reazione degli insegnanti a fronte di richieste oggettivamente irrealistiche ${ }^{48}$, come quella di far insegnare in lingua docenti privi di

\footnotetext{
${ }^{42}$ Sul riconoscimento del titolo: BOVO, Sandra, «Il "battesimo" dell'Università», in La Stampa, 28 marzo 1998, p. 37. Sui «corsi che...portino gli studenti obbligatoriamente ad avere esperienze scolastiche all'estero»: R. S., "Quasi 2 miliardi all'Università», in La Stampa 12 aprile 1998, p. 35.

${ }^{43}$ Come indicato sul sito dell'Università della Valle d'Aosta: URL: < www.univda.it > [consultato il 31 maggio 2018].

${ }^{44}$ I programmi delle singole discipline, sempre reperibili sul sito dell'Università, prevedono un numero di testi in lingua francese estremamente ridotto e ancor più rari sono i corsi per i quali è indicato l'uso veicolare della lingua francese.

${ }^{45}$ Sul rapporto tra francese e franco-provenzale nell'ultimo secolo: CELI, Alessandro, La "langue du coeur": metamorfosi e usi politici di Francese e franco-provenzale in Valle d'Aosta (1930-2012), in DI GIACOMO, Michelangela, DI NUNZIO, Novella, GORI, Annarita, ZANTEDESCHI, Francesca (a cura di), Piccole tessere di un grande mosaico. Nuove prospettive dei regional studies, Roma, Aracne, 2015, pp. 345-361.

${ }^{46}$ Legge regionale 3 agosto $2016 \mathrm{n}$. 18 (Bollettino Ufficiale, 23 agosto 2016).

${ }^{47}$ Ulteriore costante nelle vicende scolastiche degli ultimi decenni, il riferimento alle sperimentazioni del Trentino da parte dell'Amministrazione regionale.

${ }^{48}$ La legge 18/2005 ha imposto l'insegnamento in lingua inglese e francese a personale che non era stato formato in tal senso. A fronte della richiesta di ritardare di qualche anno l'applicazione della norma per consentire l'adeguata preparazione degli insegnanti, la Sovrintendenza ha scelto di mantenere un atteggiamento rigido, che ha portato a dure prese di posizione, oltre che dei sindacati, anche di numerosi
} 
preparazione specifica ${ }^{49}$, sia le esitazioni e i ritardi dovuti al quadro politico sempre più incerto a livello locale.

Rimangono, infatti, presenti le difficoltà dovute alla mancanza di coordinamento tra il bilinguismo nella Scuola primaria, basato sul sistema del 50\% della didattica in italiano e $50 \%$ in francese, e nella Scuola Media, basato sui progetti in compresenza tra insegnanti di discipline diverse, nonché l'assenza di una proposta coerente per la Scuola secondaria di secondo grado, che solo nel 2016 ha visto il primo tentativo di applicazione generalizzata del bilinguismo con il francese quale lingua strumentale.

\section{In conclusione}

Le complesse vicende della Scuola valdostana negli ultimi settant'anni consentono di identificare alcune costanti che informano l'intero periodo. La prima di esse è la dialettica tra Stato e Regione, che si esplicita nei numerosi tentativi degli organi statali di riappropriarsi delle competenze concesse alla Regione dallo Statuto di autonomia e dalle norme precedenti. La seconda è la stretta relazione tra provvedimenti legislativi e contingenze elettorali: nel 1965, 1968, 1975 e 1993 le norme regionali che modificarono lo stato giuridico o economico dei docenti giunsero a pochi mesi o a poche settimane dal voto, rivelando l'attenzione per il consenso dei docenti da parte dei consiglieri regionali. Un terzo elemento è costituito dal dibattito sull'uso del francese quale lingua di insegnamento, un problema di fatto irrisolto fino a oggi, quando risulta ulteriormente aggravato dall'introduzione dell'insegnamento CLIL in lingua inglese ${ }^{50}$. Si tratta come già detto - di un problema che deriva dalla valenza ideologica assegnata alla lingua storica della Valle nel sistema scolastico del Dopoguerra. Il desiderio di recuperare una francofonia gravemente compromessa durante la dittatura fascista divenne molto presto la bandiera ideologica di una parte politica, che non esitò ad imporla per via legislativa quando si ritrovò, per un quarantennio, alla guida della Regione. Tale scelta si rivelò due volte dannosa: dapprima perché trasformò una caratteristica culturale, in origine propria alla maggior parte della popolazione, in un elemento di riconoscimento esclusivo di un partito, provocando così la

collegi dei docenti, che hanno messo in evidenza l'impraticabilità delle scelte imposte dall'Amministrazione.

${ }^{49}$ Pur avendo superato l'esame di piena conoscenza della lingua francese, necessario per essere assunti dall'Amministrazione regionale ai sensi della legge 8 marzo 1993 n.12, la maggior parte dei docenti in servizio non ha mai usufruito di un aggiornamento disciplinare specifico in lingua né di formazione specifica durante il proprio percorso universitario, svolto in Italia per la maggior parte di essi, dato che i progetti per una loro formazione obbligatoria presso Università francofone - presenti fin dai dibattiti del 1945 - non superarono mai lo stadio delle buone intenzioni. Imporre a un docente di storia dell'arte formato in un'università italofona di svolgere in lingua francese la propria disciplina o a uno di scienze di farlo in inglese risulta, quindi, assolutamente velleitario.

${ }^{50}$ Introdotta dalla legge regionale 18/2016 come applicazione locale della legge 107/2015. 
reazione di quanti non ne condividevano gli ideali. Per un perverso, ma comprensibile, gioco delle parti questo favorì implicitamente il perdurare di posizioni che derivavano direttamente dalla propaganda e dalle politiche del Ventennio, anche da parte di partiti e sindacati che si proclamavano antifascisti, tanto che oggi la Cgil esprime le posizioni più critiche verso la presenza del francese nelle scuole valdostane. In secondo luogo, la scelta della via normativa per raggiungere l'obiettivo della rifrancesizzazione contribuì a burocratizzare la difesa e la promozione di questa lingua, che perse in tal modo l'aspetto ideale attribuitogli a partire dall'Ottocento, per trasformarsi nel sentire comune in un adempimento necessario, ma sempre più distante dalla realtà della società valdostana.

Proprio questa contraddizione ha contraddistinto e contraddistingue ancora oggi le politiche scolastiche regionali: accanto a una normativa che definisce un sistema di istruzione per molti versi all'avanguardia grazie alla capillare diffusione della scuola dell'infanzia, all'introduzione precoce del plurilinguismo, all'applicazione di modelli didattico-pedagogici innovativi, si constatano la mancata applicazione di molte delle prescrizioni di legge e un generale impoverimento del sistema scolastico. Questa situazione deriva innanzitutto dal mancato controllo da parte degli organi preposti, per timore di suscitare nel corpo insegnante reazioni negative, dalle pesanti conseguenze in termini di consenso elettorale, nonché dal perdurare di un approccio didattico all'insegnamento del francese che privilegia la conoscenza della grammatica a scapito dell'uso della lingua. Poco è stato, invece, fatto per superare l'identificazione tra sostegno del francese e appartenenza politica, cosicché l'apprendimento di questa lingua, anziché essere considerata un'importante opportunità di comunicazione con una rilevante parte del mondo, ha continuato a essere vista come espressione di appartenenza partitica. Si è così fornito un vero e proprio alibi a quanti non intendono applicare il dettato statutario nella Scuola e continuano a frapporre problemi ed esigenze, talvolta reali, talvolta ipotetici, allo sviluppo di una didattica incentrata sulle lingue vive. Questi elementi rappresentano, oggi, il principale ostacolo alla soluzione del problema dell'insegnamento in lingua francese per la Valle d'Aosta. 


\section{L'AUTORE}

Alessandro CELI ha conseguito il dottorato di ricerca in storia contemporanea all'Università di Udine e il diploma di archivista paleografo all'Archivio di Stato di Torino. Docente di lettere classiche nei licei, è cultore della materia presso l'Università della Valle d'Aosta, aderente a numerose associazioni e società culturali (SISSCO, Académie Saint-Anselme, Sabaudian Studies, ...) e componente del comitato di redazione di alcune riviste («Progressus», «Bulletin de l'Académie Saint-Anselme», «Le Flambò»). Negli ultimi dieci anni ha pubblicato due monografie e oltre trenta articoli dedicati alla storia della Valle d'Aosta e delle Alpi occidentali. Dal 2010 è presidente della Fondation Emile Chanoux, Istituto di studi federalisti e regionalisti di Aosta.

URL: < http://www.studistorici.com/progett/autori/\#Celi > 